THE ROSAT MISSION

\title{
J. Trümper
}

Max-Planck-Institut für Physik und Astrophysik, Institut für Extraterrestrische Physik

8046 Garching, W.-Germany

ABSTRACT. The scientific payload of ROSAT consists of a $83 \mathrm{~cm} \mathrm{X-ray}$ telescope $(6-100 \AA)$ and a $60 \mathrm{~cm}$ XUV-telescope $(60-300 \AA)$ which are looking parallel. An important objective of the mission is to perform the first all-sky survey with imaging $X$-ray telescopes providing an improvement in sensitivity by several orders of magnitude compared with previous surveys. A large number of new X-ray sources $\left(\sim 10^{3}\right)$ is expected to be discovered and located with an accuracy of 1 arcmin or better, depending on source strength. The sources discovered will represent almost all types of astronomical objects, ranging from nearby normal stars to distant quasars.

After completion of the sky survey which will take half a year, the instruments will be used for detailed investigations of selected sources with respect to spatial structures, spectra and time variability. In this pointing mode, which will be open for guest observers, ROSAT is expected to provide substantial improvements over the imaging instruments of the Einstein observatory.

\section{INTRODUCTION}

During the last 20 years X-ray astronomy has become one of the major disciplines in the exploration of our universe. A large variety of phenomena have been discovered in this area which comprise almost all kinds of astronomical objects - from the nearby stars to the most distant quasars at the edge of the known universe. In many cases, X-ray emission is the primary and dominating emission process ( $X$-ray binaries with neutron stars or black holes, cooling neutron stars, hot gas in clusters of galaxies etc.), in other objects, the observation of X-rays gives very important complementary information (e.g. stars, degenerate stars, active galaxies and quasars). In X-rays we see the "hot universe", explosive phenomena, large concentrations of relativistic electrons in magnetic or intense radiation fields. It is clear today that these high energy phenomena play a very important role in the evolution of matter in our universe. 


\section{ROSAT X-RAY TELESCOPE}

ROSAT is a free flying satellite to be launched by a Delta II rocket in early 1990. Its anticipated lifetime is 3 years. The scientific payload includes two instruments, a large X-ray telescope $(6-100 \AA)$ and a smaller XUV-telescope $(60-300 \AA)$ which are oriented in parallel. In this talk I'II concentrate on the $\mathrm{X}$-ray aspects.

The ROSAT X-ray telescope consists of a fourfold nested mirror system with $83 \mathrm{~cm}$ aperture and three focal instruments. Two of them will be position-sensitive proportional counters (PSPC, $6-100 \AA, 0.1-2 \mathrm{keV}$ ) having a field of view of $2 \%$. In the pointing mode the angular resolution will be 30". The positions of point sources may be determined with higher accuracy (to $\sim 10$ arcsec). The spectral resolution of the PSPC's will be $45 \%$ FWHM at $1 \mathrm{keV}$. Both PSPC's are being developed and built by MPE Garching. The third focal instrument will be a high resolution imager (HRI, 3") which is an improved version of the corresponding EINSTEIN instrument, provided by SAO/NASA. The X-ray mirror system will have a half power width of $3 "$. It is manufactured by Carl Zeiss Company in Oberkochen near Stuttgart. The calibration of mirrors and the complete X-ray telescope is done in the $130 \mathrm{~m}$ X-ray test facility of MPE Garching.

\section{THE ALL SKY X-RAY SURVEY}

A primary objective of the mission is to perform the first all-sky survey with an imaging $X$-ray telescope which will lead to an improvement in sensitivity by several orders of magnitude compared with previous "counter" surveys.

The sky survey is performed by continuously scanning great circles perpendicular to the earth-sun-line. This will result in full sky coverage in half a year. The scan of one great circle takes one orbit and thereby avoids earth occultations. At the ecliptic equator a particular source is scanned $~ 30$ times during two consecutive days with an integral exposure of $\sim 600 \mathrm{sec}$. The corresponding survey sensitivity $(5 \sigma)$ is $2 \times 10^{-13}$ $\mathrm{erg} / \mathrm{cm}^{2} \mathrm{~s}(0.1-2 \mathrm{keV})$. The ecliptic pole is crossed during every orbit which results in $\sim 60.000 \mathrm{~s}$ of total observation time and a sensitivity of $1.5 \times 10^{-}$ $14 \mathrm{erg} / \mathrm{cm}^{2} \mathrm{~s}$. We note that this latter figure is close to that of the Einstein deep surveys.

The sky survey will be done with the PSPC only because of its sensitivity and its relatively large field of view ( $2^{\circ}$ circular). The spectral resolution of the instrument allows to distinguish four "colour" bands. Positions will be accurate to 1 arcmin ( $90 \%$ confidence radius) for point sources near the sensitivity limit $S_{\min }$. For stronger sources the position determinations will be more accurate due to the better photon statistics. It 
is expected that for sources at $\sim 10 \mathrm{~S}_{\min }$ a limiting resolution of 0.5 to 0.1 arcmin ( $90 \%$ confidence radius) will be reached depending on the final instrument and spacecraft characteristics.

The most important aspect of the ROSAT star survey is that it will yield large unbiased samples of various classes of sources which are detected in a homogeneous and systematic way.

\section{THE POINTED OBSERVATIONS}

The second main objective of the ROSAT mission is to perform detailed investigations of selected sources. These will be carried out in the pointing mode and will improve our understanding of spatial structures, spectra and time variability of X-ray sources. In this mode the ROSAT Xray telescope is expected to provide a substantial improvement over the imaging instruments of the EINSTEIN-observatory, particularly in terms of sensitivity (factor $\sim 5$ to 10), spectral resolution (PSPC versus IPC factor 3) and angular resolution (factor $\sim 3$ both for the PSPC and the HRI). In addition, the XUV telescope will allow the observations to be extended to long wavelengths. In the pointing mode there will be four "X-ray colours" and three "XUV colours".

\section{THE ROSAT SATELLITE AND GROUND OPERATIONS}

ROSAT is a three-axis stabilised satellite with CCD-type star trackers (two for the X-ray telescope, one for the XUV telescope) and attitude control by momentum wheels which are desaturated by magnetic torquing. Two tape recorders are used for on-board data storage. The satellite which has a mass of more than 2,5 tons will be launched by a Delta II into an orbit of $\sim 580 \mathrm{~km}$ height and $57^{\circ}$ inclination. Mission control will be performed by the German Space Operations Center (GSOC) at Weilheim near Munich from where the data will be sent to the ROSAT science operation center at MPE Garching which is equipped with a dedicated VAX 8600 facility and a Microvax cluster.

The technical and scientific quicklook activities will be placed at GSOC and MPE as well. Date obtained during the pointed programme will be routed directly to the ROSAT data centers in the US and UK.

\section{SCIENTIFIC DATA RIGHTS}

The analysis of the ROSAT all sky surveys will be the responsibility of MPE and of the UK WFC consortium for the X-ray data and for the XUV data, respectively. 
The pointed programme will be devoted entirely to a guest observer programme. The first call for proposals will be issued one year before launch, viz. in spring 1989, in the US, UK and Germany.

\section{CONCLUSIONS}

The ROSAT mission will provide the only X-ray telescope in space during the early 1990's. It will be evident from the preceding discussion, that because of its extended waveband coverage, its sensitivity, and its spectral resolution, ROSAT will provide the scope for a wide range of novel investigations on known astrophysical objects as well as an exciting capability for new astrophysical discoveries. This is true for the pointed observations, but also for the all-sky survey which will have a great impact on the work in other regions of the electromagnetic spectrum. Last, but not least, the survey will help to guide the observations of followup $X$ ray astronomy missions, including in particular the large permanent $X$-ray observatories to be launched in the late 1990's, AXAF and its European complement XMM.

\section{REMARK}

A more detailed description of the project including references is given by the present author in Physica Scripta, T7, 1984. 